The use of electron-spin resonance in free-radical investigations has already been mentioned, and Prof. D. J. E. Ingram (University College, Keele) outlined how this can be used in the study of proteins, where electron trapping may occur. It may be possible to correlate denaturation of a protein with an interference in this trapping process since the electron-spin resonance signal does not disappear on warming in the normal way. However, it must be remembered that when applying electron-spin resonance to biological materials, water, for example, plays a part which is little understood.

Sulphur compounds are of much interest on account of their use as protective agents. Dr. R. Koch (Freiburg) investigated more than a hundred compounds by electron-spin resonance, but no simple correlations between structure and function can be seen at the present time. The radical nature is considered to be locelized on the sulphur atom, and Dr. P. Alexander (Chester Beatty Research Institute, London) and Mr. M. G. Ormerod (Royal Military College of Science, Shrivenham) provided evidence that in the case of nucleoprotein protected by cysteamine, such localization can occur, minimizing the damage. There seems little doubt that the radicals produced on irradiation of keratin are cystine-like (Dr. A. Redhardt and Prof. B. Rajewsky, Max Planck Institute for Biophysics, Frankfurt), but unaccountably, cysteine and related compounds do not appear to protect by a factor of more than two in a system such as yeast, when judged by quenching of electron-spin resonance signals (Dr. B. Smaller, Argonne National Laboratory, Illinois).

In general, it appears that dry solids are more sensitive than wet solids or solutions, and Dr. R. A. Braams (Physical Laboratory, University of Utrecht) reported a ratio of three between dry and wet tendon. Dr. Robins and Prof. Butler found solid trypsin to be more sensitive than its solutions, possibly due to the self-annealing processes in the presence of water. The last-mentioned authors also reported that the radiosensitivity of trypsin is modified by the medium from which it is evaporated and a low $p H$ leads to a highly sensitive state, possibly by aggregation. It is significant that at high linear energy transfer Brustad obtained target sizes in excess of the accepted molecular weight for some enzymes; this also raises the question of energy transfer in the solid state. The effect of added materials depends on their being intimately mixed with the substrate, and the state of the solid is thus again seen to be important.

Two papers were presented dealing particularly with chemical changes on irradiation. Dr. W. M. Garrison (Crocker Laboratory, Lawrence Radiation Laboratory, Berkeley) found good evidence for the conversion of the - $\mathrm{NH}-\mathrm{CH}-$ configuration in amino-reids into keto-acids, and as little as $10^{-8}$ mole can be detected as derivatives. Dr. H. A. Dieu (Laboratoire de Chimie Physique, Université de Liège) pointed out that irradiation of ovalbumin leads to a more ready attack by proteolytic enzymes, and an 'unmasking' of sulphydryl groups with the heaviest doses. Moreover, since the rate of subsequent attack by pepsin depends on the dose, and that by trypsin does not, it is probable that the aromatic amino-acids have been preferentially destroyed. In the myoglobin molecule it appears that possibly the leucine residues are the foci of damage, in particular those in more-exposed parts of the molecule, and Mr. C. C. F. Blake and Dr. D. C. Phillips (Davy Faraday Laboratory, Royal Institution, London) provided evidence for this in which the effect of radiation was studied directly by $\mathrm{X}$-ray crystallography.

In summing up, Dr. L. H. Gray (Radiobiology Unit, Mount Vernon Hospital, London) stressed that physical theories, such as that of Prof. U. Fano, must be cautiously applied to biological systems, bearing in mind their complexity and such unknown factors as water content. It appears that the degree of crystallinity of the solid phase may be of great importance, and electron-spin resonance has helped to emphasize this. Concerning the role of oxygen, the accessibility of this gas may be a factor that has not received enough attention hitherto.

Finally, I was struck by the different interpretations that were placed on electron-spin resonance signals, and feel that this useful technique needs to be used with reserve when drawing conclusions.

A. B. Rosins

\title{
BACTERIA OF THE INTESTINE
}

T HE summer conference of the Society for Applied Bacteriology was held at Wye College, Ashford, Kent, during July 11-13, and included a symposium on "Bacteria of the Intestine".

Dr. H. Williams Smith, in his paper entitled "The bacterial flora of the fæces of animals with particular reference to its development in the young", described experiments on the development of normal flora in healthy animals. Rectal swabs were shaken in phosphate buffer, and after passing the suspension through muslin, colony counts of Escherichia coli, staphylococci and Clostridium welchii were made.

Calves, lambs, piglets and a baby were examined daily for 2-3 weeks, weekly for three weeks, monthly for nine months and twelve months (baby). One rabbit was examined weekly.

In the calf, $E$. coli, streptococei and $C l$. welchit predominated on the first day, but bacteroides domination began at two days and these organisms rapidly multiplied up to fourteen days, when lactobacilli followed. Counts slowly dropped to $10^{7}$ $10^{6} / \mathrm{gm}$. of fæces after thirty weeks.

Similar results were obtained from lambs and piglets, except that streptococci dominated later with E. coli in lambs, and the piglet flora was dominated ultimately by lactobacilli. Results for the baby were similar also, with counts of $10^{11}$ for $E$. coli at first, followed by a bacteroides count of $10^{10} ; \mathrm{Cl}$. welchii rose sharply at twenty-five weeks when the child started eating solid food. The counts remained high after a year; this was different from the animals. Staph. aureus, $10^{2}-10^{6} / \mathrm{gm}$, was frequently found in the baby but not in the animals. The rabbit showed a constant bacteroides count of $10^{9}$; the lactobacilli and streptococcal counts were $10^{4}$ and $10^{5}$. Gram-negative anaerobic cocci up to $10^{8}-10^{9}$, and yeasts in older calves, were also found. There was a constant population of $E$. coli but frequent changes in phage types. 
Adult animals, including cats and dogs, showed a high count of $C l$. welchii which varied in degree according to the diet, being alternately high and low when fed on meat or bread. Similarly, if pigs were starved and fed alternately the count went down and up accordingly. The floras of adult guinea pigs were dominated by lactobacilli.

Dr. H. Haenel ("Micro-ecological rules in the compcsition of the intestinal flora of man") said that before birth the baby was germ-free, at birth infection was acquired from the mother's vagina and fæces, and after 3-4 days lactobacilli predominated. The effect of the mother's milk was to replace aerobic lactobacilli by Lactobacillus bifidus and to depress clostridia but not $\boldsymbol{E}$. coli. After weaning, $L$. bifidus stayed at a high level, but aerobic lactobacilli and clostridia returned later. Similar results were obtained for poorly developed children in hospital. Factors ruling the flora were easily disturbed; when stools were stored the lactobacilli diminished and streptococci increased and under abnormal conditions there was a reduction of lactobacilli and an increase in proteolytic organisms.

Antibiotics such as terramycin abolished $L$. bifidus and there was an increase in streptococci and aerobic lactobacilli, with an occasional predominance of Proteus; the normal flora returned quickly when the antibiotic was stopped. Under antibiotics salmonellæ disappeared but they could return later; they sometimes shared the normal flora without disturbance. After gastrectomy, the anaerobic lactobacilli disappeared, $E$. coli counts became higher than normal ; enterococci, clostridia and other organisms increased also and the large intestine showed high counts. In the adult the flora was stable. Normally the small intestine was nearly sterile, and the large intestine showed mostly anaerobic lactobacilli. Under abnormal conditions there could be a predominance of $E$. coli in the small and no anaerobic lactobacilli in the large intestine; the flora might be the same throughout the intestine with anaerobic lactobacilli absent.

Dr. Anne Dickinson and Dr. G. Mocquot ("Studies on the bacterial flora in the alimentary tract of pigs: 1 , Enterobacteriaceae and other Gram-negative bacteria"), observed pigs of $20-100 \mathrm{kgm}$. in individual pens; the diet was supplemented with antibiotics in some animals. The object was to identify Enterobacteriaceae and other Gram-negative flora isolated on deoxycholate citrate agar after 24-28 hr. at $37^{\circ} \mathrm{C}$. Details were given of ten groups classified mainly on morphology, colonial appearance, motility, whether mucoid or not, glucose agar stab cultures, lactose fermentation, growth at $45^{\circ} \mathrm{C}$., indole, lysine decarboxylase, urea hydrolysis, production of hydrogen sulphide, liquefaction of gelatine, and ornithine and ammonia. There was no change in the distribution of types under antibiotic feeding. Group 1 usually dominated and the other groups varied for no apparent reason.

Drs. P. Raibaud, M. Caulet, J. V. Galpin and G. Mocquot ("2. Streptococci : selective enumeration and differentiation of the dominant groups") described the isolation of 415 strains of group D streptococci from cæcal and frcal swabs, using their own medium and also those described by Barnes, Slanetz and Kjellander; thirty-eight types could be distinguished by fermentation reactions differing in at least one carbohydrate. Urea hydrolysis was used for differentiation and the results were regarded as of taxonomic significance. The predominant strepto- cocci were related to Streptococcus bovis or Strep. equinus, but the classification of these organisms is at present extremely confused.

Dr. M. Lev's paper on "Germ-free animals and their use in elucidating the action of the gut flora on the host" was read by Dr. Cuthbert. Using an apparatus consisting of a nylon tube over a frame, all of which could be autoclaved, chickens and turkeys were hatched from washed and sterilized eggs and the young mammals, monkeys and guinea pigs removed by cæsarean section. Sterilized foods with and without antibiotics were given through tubes, and the effect on the germ-free birds and animals noted. Normal chicken fæces fed to germ-free chicks depressed growth, but feeding penicillin depressed $C l$. welchii and stimulated growth. Feeding $C l$. welchii to germ-free chicks depressed growth, but the effect was partly reversed by penicillin in the diet; growth was not increased when germ-free animals were given penicillin. Toxins of $\mathrm{Cl}$. welchii caused the gut to thicken and this was prevented by penicillin. Feeding lactobacilli, streptococci and $\boldsymbol{E}$. coli to germ-free creatures had no effect, but the addition of $\mathrm{Cl}$. welchii depressed growth, which again was partly reversed by penicillin.

Deficiency in vitamin A led to infection, and gross deficiency caused death; lesions could be seen in the germ-free animals. Germ-free animals on a vitamin $\mathrm{K}$-deficient diet suffered prolonged gut bleeding and the blood had no clotting power. Menedian (four vitamins) did not restore the clotting power, and it is suggested, therefore, that gut bacteria are needed to convert the menedian to some form which can be used.

Entamoeba histolytica did not attack the germ-free gut but survived for a few days only when coliaerogenes were present; they did not survive when autoclaved cæcal contents were fed. There appeared to be complex factors involved in harmless organisms promoting a lethal effect in contact with pathogens. Shigella flexneri did not attack the normal guinea pig, but killed the germ-free animals; this was prevented by prior feeding with $\boldsymbol{E}$. coli, non-colicine forming and not demonstrably toxic in vitro.

Germ-free animals were equally affected by hæmorrhagic shock caused by the endotoxins from gut Gram-negative flora. Germ-free chicks after five weeks exposure to conventional conditions suffered severe setbacks. Tests are needed on synthetic diets.

Dr. Joan Taylor, describing the "Host specificity and enteropathogenicity of Escherichia coli", said that the typing of pathogenic $E$. coli was based on ' $O$ ' (somatic), ' $K$ ' (surface) and ' $H$ ' (flagellar) antigens. Infantile diarrhcea was due to types 0111, 055 and 026 mainly, which were found in all parts of the world. 0128 and 0127 could be found in the United Kingdom and United States respectively, but they were now beginning to cross-spread.

Epidemiological correlation confirmed the role of these organisms in infantile diarrhoea. With the three major types, it was possible to follow the rise in numbers until diarrhoea occurred, beginning when there was a pure culture and ending when other organisms were re-established. But there were similar occurrences with no symptoms. Volunteer feeding had confirmed the pathogenicity of 0111 in students, prisoners and a baby, and of 055 more feebly in prisoners.

Calf scours was related to $E$. coli, particularly 078 , which was widely distributed, and may become 
septicæmic. Swine œedema due to 0138 and 0139 was frequent in the United States, United Kingdom and Canada. Enteritis in lambs due to type 078 occurred in the United Kingdom and Australia; hen septicæmia was caused by type 02 .

There was little information about the occurrence of these organisms in healthy animals, but the human types $026,055,0111$ were found in eats, dogs, and kittens without symptoms. Surveys suggested limited distribution in different species and disease in some only.

Infection experiments were carried out on alternate loops of sterile gut, in anæsthetized rabbits; the rabbits were killed $24 \mathrm{hr}$. later by injection of air. Inflammatory reactions with exudations occurred in the infected loops, but non-infected loops were sterile and normal. Many strains from human babies, also chicks, water, calf and swine strains, gave no reaction, although some calf-strains were positive. Living cells were necessary for infection.

Dr. J. G. Collee, Miss J. A. Knowlden and Dr. Betty Hobbs, in their paper on "Studies on the growth, sporulation and carriage of Clostridium welchii with special reference to food poisoning strains", observed that typical $C l$. welchii type $A$ strains were completely $\beta$-hæmolytic, and that strains were called non-hæmolytic if the hæmolysis was not complete. Food poisoning strains were non-hæmolytic and resisted $100^{\circ} \mathrm{C}$. for $1 \mathrm{hr}$. $80 / 80$ samples of fæces yielded $\mathrm{Cl}$. welchii. 39/50 (78 per cent) stool samples from students gave non-hæmolytic strains, but spores from three $(6$ per cent) strains only were remarkably heat-resistant, as demonstrated by tests on fæces and tests on sporing cultures. Previous reports had given 2 per cent as the average carrier-rate of non-hæmolytic heatresistant types in normal people; hospital personnel had shown 20 per cent or more. Heat-resistance tests for spores in stock strains revealed the protective effect of cooked meat, which was most apparent with heat-resistant strains. Preliminary findings indicated that the heat-resistant property of typical food-poisoning strains was genetically determined and was not primarily dependent on the presence of large numbers of spores.

Food-poisoning strains seldom produced spores in cooked foods and it was suggested that food should be streaked on neomycin blood agar for nonhæmolytic anaerobic colonies, which should be picked off, grown in Ellner's sporulation medium and the heat-resistance tested. It should be noted that hæmolysis on blood agar was not entirely con. sistent and spore yields from Ellner's medium were not reliable. Confirmation was given by slide agglutination with the thirteen antisera available; serological cross-reactions and untypable strains still occurred occasionally. It was possible to type 75 per cent of 298 strains submitted during 1960 . 1961 .

An unusual growth pattern at $50^{\circ} \mathrm{C}$., the Phonix phenomenon, was observed with both heat-sensitive and heat-resistant strains; there was no difference between the growth curves of food-poisoning and non-food-poisoning hæmolytic strains over the normal range of temperature, and both could grow under nearly similar conditions, which accounts for occasional outbreaks caused by the heat-sensitive $\beta$-hæmolytic types. These strains were unlikely to survive cooking, but may be protected mechanically by lack of heat penetration or may be introduced and find a favourable anaerobic environment during the cooling period.
It was stressed that the relationship of these observations to the bulk cooking of meat and its known association with $\mathrm{Cl}$. welchii poisoning of food merited further investigation.

Dr. Betty Hobbs, describing "The public health significance of Salmonella carriers in livestock and birds", considered the carriage of salmonellæ by animals and birds in relation to the contamination of foods provided for human consumption; human carriers now appeared to be less important than animal carriers. Particular attention was directed to poultry and egg products and to the contamination of boneless and carcass meats, horse meat and pet food. A parallel was shown between Salmonellae isolated from abattoirs and in local populations.

It was suggested that improved conditions and speed of transport for animals passing from farms to abattoirs and more care and attention over hygiene in processing may reduce the spread of infection among animals and the spread of contamination on carcass meat; also, that the pasteurization of egg products and pelleting of animal foodstuffs, both of which eliminated Salmonellae, would help markedly to reduce the introduction of Salmonellae into bakeries and farms. The increase in salmonellosis due to exogenous types was most probably due to the introduction of egg products, meat, and now coconut contarninated with these types.

Drs. R. Buttiaux and D. A. A. Mossel ("The significance of organisms of supposed frcal origin in food and water") suggested that the enumeration of organisms indicative of freal contamination was necessary because: (a) direct tests for pathogens were difficult and statistically uncertain, and $(b)$ repeated absence of indicators gave virtual certainty of absence of fæcal contamination, and also $(c)$ guaranteed absence of non-pathogenic fæcal contamination. Human indicator organisms had been preferred hitherto but animal indicators might be equally or more important.

The test should be quantitative and foods classified as stable, that is, dried, or perishable, raw or heated. The indicator organisms should be frequent in the gut and scarce outside, but they must be able to survive outside the enteric environment.

$E$. coli $I$ was common in fæces but not outside. $E$. coli $I I$ was fairly common in frees but occurred elsewhere also. Klebsiella, non-motile and ureasepositive, were frequent in human stools, also in swine stools but seldom elsewhere. Lancefield Group $D$ streptococci were common in animals but little was known about their survival in the environment. L. bifidus and bacteroides were unsuitable.

Dr. J. H. McCoy, introducing the discussion, empha. sized the fact that examination of water was chiefly concerned with measures against typhoid fever, dysentery and cholera and none of the bacteria responsible was carried by animals; thus interest was centred on human contamination. The survival of $E$. coli in water meant recent pollution; if these organisms were absent there had been time for pathogens to die, as the survival-rate of $E$. coli was similar to that of pathogens. The treatment of sewage aimed to reduce nuisance, not bacteria; Salmonellae could be isolated easily from treated sewage. In food the organisms had opportunity for growth, hence numbers of fæeal organisms at time of delivery bore no relation to the degree of original contamination. In untreated food the presence of intestinal organisms led to an assessment of cleanliness and not to safety; therefore there should be direct examination for pathogens also. 
Dr. N. P. Burman, who continued the discussion, showed tables which gave the optimum growth temperature characteristics of coli-aerogenes from various sources and also the coli-aerogenes and streptococcal flora of shallow wells and mains. IMVIC types other than $E$. coli could be found in a variety of places, for example Wilson's irregular type 6.

Coliforms were stimulated by copper treatment, and $C l$. welchii could be found in chlorinated water supplies. Streptococei found in river waters were mostly animal types, although many strains on membrane filters at $37^{\circ} \mathrm{C}$. were untypable; these were eliminated if incubation at $37^{\circ} \mathrm{C}$. was exchanged for $45^{\circ} \mathrm{C}$. after $4 \mathrm{hr}$.

In more general discussion, Dr. Blanche Koelensmid and others expressed satisfaction at the authoritative indication that fæcal bacteria in foods cannot be regarded with the same significance as in water.

\section{VITAMIN $B_{12}$ AND INTRINSIC FACTOR}

$\mathrm{A}$ BOUT 150 leading workers from forty-five laboratories in fifteen different countries attended the second European Symposium on "Vitamin B B $_{12}$ and Intrinsic Factor", organized by Prof. J. Kühnau and Drs. W. Friedrich and H. C. Heinrich, and held in the Institute of Physiological Chemistry in the University of Hamburg during August 2-5. Prof. Kühnau, in his opening address, emphasized the importance of the newly revealed structure of vitamin $B_{18}$ coenzyme, endowing it with remarkable activity. In the first symposium at the same place five years ago a notable feature was the disclosure of the structure of vitamin $B_{12}$, the culmination of several years work by Dr. Dorothy Hodgkin et al. at Oxford. This was then the most complex molecule of which the structure had so far been elucidated. In this second symposium, the climax of years of patient work by Drs. Hodgkin and P. G. Lenhert came just in time for the disclosure of the structure of a still more complex molecule, that of a vitamin $B_{12}$ coenzyme, the form in which the vitamin appears mainly to exist in the animal and human body.

Crystals of the 5:6-dimethylbenziminazole cobamide coenzyme were grown and photographed with copper X-radiation while in contact with their mother liquor. A complete $\mathrm{X}$-ray analysis of the structure revealed some interesting differences from the structure of cyanocobalamin, which at present is the official form of the vitamin. The new unsaturated pentose previously reported to be liberated from the coenzyme by mild acid hydrolysis has now been found to be an artefact produced during the hydrolysis from ribose through which adenine is linked to the cobalt in the molecule. There may be a free valency in the coenzyme molecule that could play a vital part in metabolic reactions yet to be elucidated.

These differences in chemical composition and molecular structure between vitamin $B_{12}$ and its coenzyme are reflected in differences in their absorption spectra. H. A. Barker et al., at the University of California, where pioneer work on the coenzyme was done, found that in place of the characteristic sharp band of cyanocobalamin at $361 \mathrm{~m} \mu$ the coenzyme shows a broader band at about $380 \mathrm{~m} \mu$ and a very slight band at 340-345 mp. The fairly broad cyanocobalamin band with maxima at about 520 and 550 $\mathrm{m} \mu$ is replaced by a very broad $B_{12}$ coenzyme band with maximum at about $520 \mathrm{~m} \mu$ and a slight shoulder at about $495 \mathrm{~m} \mu$. The cyanocobalamin band at about $278 \mathrm{~m} \mu$ is replaced by an inflexion at $288 \mathrm{~m} \mu$ in alkaline solution shifting to $284 \mathrm{~m} \mu$ in acid solution and considered specific for the coenzyme. Characteristic differences in absorption spectra of purineand benzimidazole-containing coenzyme anklogues appear to depend on differences in the strength of the bond between the base and cobalt. There is evidently scope for further spectroscopic studies, including a search for isosbestic points that are so prominent in mixtures of cyano- and hydroxocobalamin, now considered to be artefacts obtained from $B_{12}$ coenzyme in the liver and other body stores. E. E. Gabbe and H. C. Heinrich (Institute of Physiological Chemistry, University of Hamburg) emphasized the importance of the freedom of $\mathrm{B}_{12}$ coenzyme from cyanide, which they investigated in experiments using radioactive preparations.

$\mathrm{B}_{12}$ coenzymes catalyse various reactions that can be used for their enzymic assays. These include conversion of glutamate to $\beta$-methylaspartate by the bacterial glutamate isomerase system, the methylmalonyl isomerase reaction and the conversion of certain glycols to deoxyaldehydes. Experiments with deuterium oxide exclude reaction mechanisms involving the intermediate formation of a free proton. These reactions have been studied in bacterial extracts but the methyl-malonyl isomerase reaction occurs in higher organisms.

These findings apply to different $B_{12}$ coenzymes, of which several have been isolated by $\mathrm{H}$. A. Barker et al. They appear to be the most abundent corrinoid compounds in various bacteria (for example, Propionibacterium) and in mammalian, including human, livers. However, D. Perlman and his colleagues at the Squibb Institute for Medical Research, New Jersey, obtained only 5,6-dimethylbenzimidazolyl cobamide coenzyme from $\mathbf{3 0}$ cultures of Streptomyces.

$\mathbf{B}_{12}$ coenzymes are highly sensitive to light and cyanide. Photolysis yields hydroxocobalamin, which the minute traces of cyanide always present in blood can partly convert to cyanocobalamin. Polarographic studies of these reactions have been carried out by $\mathrm{K}$. Bernhauer et al. at Stuttgart. While body tissues usually contein a mixture of hydroxo- and cyanocobalamins, the latter, being at present the officially accepted form of vitamin $B_{12}$, has been chosen as the standard of comparison with $B_{12}$ coenzyme by a number of investigators. M. E. Costes et al. (National Institute for Research in Dairying, Shinfield) detected no difference between the absorption of $B_{12}$ coenzyme and that of cyanocobalamin (both labelled with cobalt-58) through rat intestine in presence of rat stomach extract. Chick assays showed the coenzyme to have only 78 per cent of the activity of cyanocobalamin when administered intramuscularly, but the coenzyme appeared more effective than cyanocobalamin in restoring hatchability in depleted chick embryos. On the whole the coenzyme does not seem to have any therapeutic advantage over cyanocobalamin.

A. W. Johnson (University of Nottingham), described synthetic approaches to the corrin ring 